\title{
Self-ligation shortens chair time and compounds savings, with external bracket hygiene compared to conventional ligation: Systematic review with meta-analysis of randomized controlled trials
}

\author{
JC Voudouris $^{1 *}$, S Suri ${ }^{1}$, B Tompson ${ }^{1}$, JD Voudouris ${ }^{1}$, C Schismenos ${ }^{2}$ and J Poulos ${ }^{3}$ \\ ${ }^{1}$ University of Toronto, Canada \\ ${ }^{2} \mathrm{New}$ York University, USA \\ ${ }^{3}$ Ohio State University, USA
}

\begin{abstract}
Objective: To test if there are significant evidence-based differences in effectiveness between self-ligation (SL) and conventional-ligation (CL) brackets.

Materials and Methods: Popular clinical claims of SL were identified through a literature overview of PubMed, EMBASE, Cochrane Library, and Web of Science for the period 1965-2017. Additional hand searching of the references from retrieved articles was completed. The articles containing the inclusion criteria were qualitatively analyzed using the Cochrane risk of bias tool, and one other scale. Applicable RCTs were statistically analyzed with weighted means calculations and forest plots. RCT data that could not be synthesized with one other RCT at this time were reserved for discussion.

Results: The inclusion criteria were satisfied by a total of ten RCT studies, six of which were matched for meta-analysis of three popular clinical claims. Space closure rate, reduced incisor proclination, and the rate of mandibular alignment for SL compared to CL were not statistically significant with confidence intervals of $95 \%$. The remaining four RCTs were collectively analyzed and found no statistically significant difference in discomfort between SL and CL.
\end{abstract}

Conclusion: The null hypothesis that there are no differences between SL and CL, was not rejected due to statistically insignificant results. Additional active SL studies, and well-designed RCTs for MA are needed that includes overall treatment time. SL chair time efficiency was consistently higher versus CL.

\section{Introduction}

Why do seasoned orthodontists continue to apply selfligating brackets internationally? Self-ligation (SL) proponents and manufacturers of SL systems have made clinical claims of their effectiveness and efficiency over conventional ligation (CL), but the scientific evidence for several clinically relevant assertions appears to remain inconclusive. A few published systematic reviews (SRs), including one SR of in vitro studies, tried to address many of these questions, but there was a lack of high quality evidence available at the time of their publication, a chronic problem with the extremely high-bars to reach in SRs. Since that time, however, there has been a significant five-fold increase in the number of randomized controlled trials (RCTs) on SL compared to CL, that were suggested to be employed exclusively for meta-analysis (MA), and to prevent statistical errors [1].

Clinically, it may have been in 1935 that the first SL, single-wing bracket design was introduced, but SL for the most commonly used type of orthodontic brackets, like the siamese twin bracket design, was only developed recently within the last 18 years. This appears to be a central inflection point because it coincides with an increased frequency of use for twin SL and the associated claim of chair time efficiency. However, the number of other SL advantages began to escalate dramatically, with counter-arguments that refuted all advantages of SL. Significantly, there have also been two SL bracket mechanisms and schools of siamese twin philosophies: the interactive (ISL) or active, and the passive (PSL) as 2 different groups for SL investigations.

Few differences were found for dental-occlusal dimensions such as molar and intercanine width comparing SL with CL. No adequate, evidence-based data were available that showed SL increased maxillary and mandibular buccal bone growth, reduced root resorption, improved torque, or increased bond failure rate. Since the publication of past SRs, many RCTs comparing SL with CL have been published in the peer-reviewed literature and inclusion of these recent RCTs for MA is indicated.

The aim of this SR with MA is to evaluate whether SL brackets are more effective than CL brackets by testing hypotheses generated from several popular clinical claims including that SL shows: 1) earlier in

*Correspondence to: John Voudours (Hon) DDS, DOrth, MSc(D) University of Toronto, Discipline of Orthodontics New York University, Division of Biological Sciences AAO Milo Hellman Research Award Recipient 1988 Member Edward H Angle East Society of Orthodontists, Canada, E-mail: jvoud@me.com; jvoud@pathcom.com

Key words: benefit/cost analysis of the dental sector, overall dental health indicator, performance of dental care sytems, efficiency of a country's oral care

Received: May 05, 2018; Accepted: May 18, 2018; Published: July 04, 2018 
Voudouris JC (2018) Self-ligation shortens chair time and compounds savings, with external bracket hygiene compared to conventional ligation: Systematic review with meta-analysis of randomized controlled trials

vivo space closure, 2) reduced lower incisor proclination, 3) earlier initial mandibular incisor alignment, 4) reduced initial discomfort and 5) reduced halitosis. The additional objective is to test if SL brackets are more efficient, defined as maximizing outputs of patient-doctor time, compared to $\mathrm{CL}$ in terms of 6) reduced overall treatment time, and 7) shortened chair time. By applying a search strategy to identify RCTs, the objective is to test if SL compared to CL has any treatment advantages. The higher filter for the data uses at least two RCTs for MA to determine statistical significance [1] The null hypothesis is that high quality evidence-based data do not substantiate a significant difference between SL and CL.

\section{Methods and Materials}

This review applied RCTs for MA of the clinically relevant questions. The treatment outcomes that could be studied statistically were determined by the number of RCTs matched for MA and followed the PICOS format. Inclusion and exclusion criteria were applied a priori to select articles.

\section{Inclusion criteria}

1. RCTs were assessed with other closely comparable RCTs using the Cochrane risk of bias, for MA (one other tool was used for the characterization of the good quality studies with a scale for low, moderate, and high-risk bias).

2. Studies including SL and CL orthodontic treatment that did not discriminate for age or gender.

3. Studies that compared SL with CL appliances concerning effectiveness (i.e. chair time efficiency).

\section{Exclusion criteria}

1. Studies that did not have a comparison cohort.

2. Animal studies, in vitro, or ex-vivo studies.

3. Editorials, opinions without age, gender, language discrimination or analytical outline.

Four main electronic databases, including PubMed, EMBASE, Cochrane Library, and Web of Science, were reviewed comparing SL to CL studies from 1965 until 2017. Search strategies and keywords were employed for each database (Appendix 1). The literature overview was used to identify the prevalence of SL clinical claims qualitatively by listing them in customized forms by two reviewers (U.V., W.X.).

Articles to be included were independently read, reviewed and discussed without language discrimination. Inclusion was based on consensus agreement by at least 2 of 3 investigators (U.V., W.X., Y.Z.) using independent search and custom data abstraction forms piloted on RCT, cohort and cross-sectional studies. Article references were also hand-searched. Quality investigations were included that had well-conducted statistical analysis (independent of a low, moderate or high risk of bias) and a statistics expert was consulted during statistical analysis. If there was disagreement, a third reviewer (U.V.) was used to reach consensus. Grey literature was evaluated for an overview but in the end peer-reviewed articles were included.

Independent quality assessment for bias (Appendix 2a,b), was conducted for the included, peer-reviewed studies using the Cochrane risk of bias assessment tool specific for RCTs and the modified Newcastle-Ottawa scale for also Non-Randomized Trials, (NRT's) by 2 researchers (W.X., Y.Z.). A third investigator (U.V.) was consulted in areas of dispute to form a consensus. Seven criteria details in the Cochrane tool were applied separately for the RCTs. Low (+), high (-) or unclear (?) risk of bias was given to each criterion to determine overall level of bias. Qualitative assessment of each peer-reviewed study not only encompassed risk of bias, but also contained a detailed characteristics evaluation for each of the included investigations.

Quantitative assessment was then made using the RCTs that could be matched with at minimum, another RCT study for a MA to be performed. A threshold level of a low or moderate level of bias for each quality RCT was deemed to be acceptable for MA. If a MA could not be conducted using the articles included in the SR, it was not considered quantitative evidence. Additionally, a sensitivity analysis was performed, where necessary, with regard to qualitative analysis, risk of bias, and publication status.

Single good quality RCTs that met the inclusion criteria, but that could not be matched, and currently could not undergo MA were not included in the statistical results. Rather, they were reserved for discussion because of their potential to possibly guide the designs of future RCTs needed for MA [1].

\section{Meta-analysis}

A MA was performed to synthesize comparable RCT data for each clinical claim of interest using the metaphor [2] package in R software (MATLAB version 8.2, Mathworks, Natick, Massachusetts, United States). Heterogeneity was assessed for the included investigations. A fixed-effects model was used with less heterogeneous results ( $\mathrm{I}^{2}$ statistics $<75 \%)$. A random effects model was used for more heterogeneous results ( $\mathrm{I}^{2}$ statistics $>75 \%$ ). Forest plots were constructed using weighted mean differences only from RCTs. If high quality RCT investigations with lowest possible bias could not be found for a specific claim, forest plots were not constructed. Dichotomous data was assembled with the use of odds ratios. Funnel plots were used to assess publication bias.

\section{Results}

The electronic search and overview of the orthodontic literature identified 236 studies for prevalence of clinical claims. The claims were divided into two groups, primary questions 1-7 and secondary questions 8-19 (Table 1), toward the preparation of the MA. From the 236 articles, 35 met the inclusion criteria (Table 2, 22 RCTs and 13 NRTs), including one from hand searching (Figure 1). Table 2 outlined the characteristics and details of each of the 35 studies including their risk of bias. The 13 NRTs were characterized and reserved for discussion.

Ultimately, 10 RCTs were found. For three relevant SL claims (in vivo space closure, incisor proclination and rate of mandibular incisor alignment in days,) a MA was performed using calculated weighted mean differences. There were two RCTs for each of three claims producing six matched RCTs and forest plots were constructed for the three relevant claims (Figures 2-4). In addition, the data of four RCTs on discomfort were synthesized for MA. The excluded studies did not provide useful data.

\section{Rate of in vivo Space Closure}

There were two in vivo studies on distal movement of canines for space closure on round wire that applied retraction forces directly on the canine hooks (Figure 2). The forest plot showed a mean difference of $0.17 \mathrm{~mm}$ for CL in the study by Burrow [3]. Compared to $0.06 \mathrm{~mm}$ for PSL in the investigation by Mezomo, et al. [4] with both studies using 28-day intervals of canine retraction. However, this small effect 
Voudouris JC (2018) Self-ligation shortens chair time and compounds savings, with external bracket hygiene compared to conventional ligation: Systematic review with meta-analysis of randomized controlled trials

Table 1. Nineteen claims of self-ligation from electronic literature overview

\section{Primary Claims}

\begin{tabular}{|c|c|c|c|}
\hline 1. Higher in vivo space closure & 3. Faster initial mandibular incisor alignment & 5. Lower overall treatment time & 7. Shortened chair time \\
\hline 2. Reduced incisor proclination & 4. Reduced initial patient discomfort & 6. Halitosis and periodontal health & \\
\hline \multicolumn{4}{|l|}{ Secondary Claims } \\
\hline 8. Improved oral hygiene, bacterial levels & 11. Greater interbracket flexibility & 14. Reduced bond failure & 17. Bracket deformation \\
\hline 9. Lower moments of rotation & 12. Reduced root resorption & 15. Clip wear & 18. Intercanine and intermolar width \\
\hline 10. Max \& mand buccal bone formation & 13. Improved torque & 16. Reduced in vitro resistance to sliding & 19. Bracket oxidization \\
\hline
\end{tabular}

\begin{tabular}{|l|l|}
\hline 12. Reduced root resorption & 15. Clip wear \\
\hline 13. Improved torque & 16. Reduced in vitro resistance to sliding \\
\hline
\end{tabular}

19. Bracket oxidization

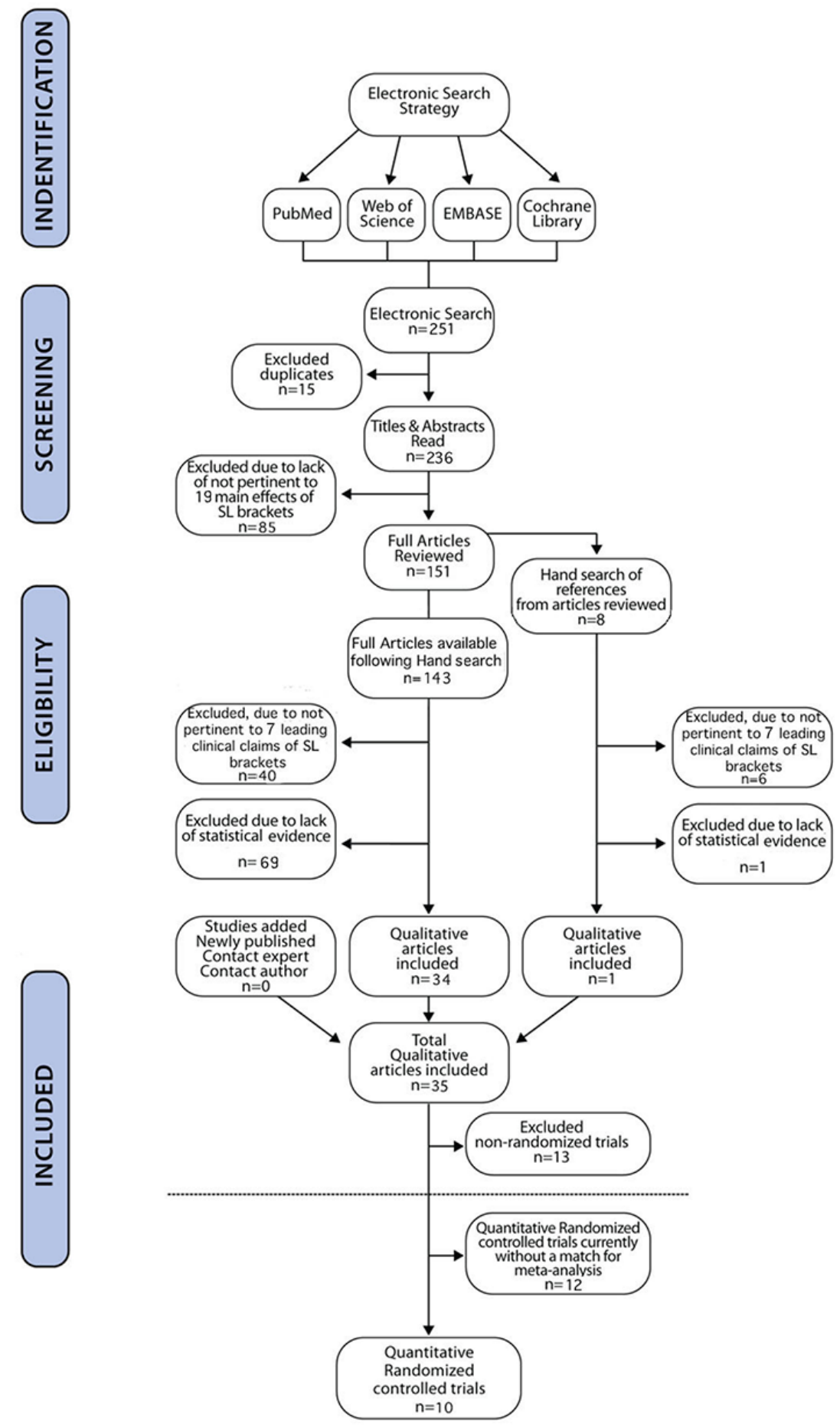

Figure 1. Flow Diagram for determining quantitative articles used 
Voudouris JC (2018) Self-ligation shortens chair time and compounds savings, with external bracket hygiene compared to conventional ligation: Systematic review with meta-analysis of randomized controlled trials

Table 2a. Characteristics of RCTs included (and other quality investigations by publication date, See Appendix 2 for qualitative assessment)

\begin{tabular}{|c|c|c|c|c|c|c|}
\hline Author & Kaygisiz et $\mathrm{al}^{46}$ & $\mathrm{O}^{\prime}$ Dwyer et $\mathrm{al}^{30}$ & Rahman et $\mathrm{al}^{26}$ & Celikoglu et $\mathrm{al}^{21}$ & Uzuner et $\mathrm{a}^{47}$ & Nalcaci et $\mathrm{al}^{45}$ \\
\hline Year & May 2015 & April 2015 & March 2015 & March 2015 & Nov 2014 & May 2014 \\
\hline Design & $\begin{array}{l}\text { Randomized controlled } \\
\text { trial }\end{array}$ & $\begin{array}{l}\text { Randomized controlled } \\
\text { trial }\end{array}$ & $\begin{array}{l}\text { Randomized } \\
\text { controlled trial }\end{array}$ & $\begin{array}{l}\text { Randomized controlled } \\
\text { trial }\end{array}$ & $\begin{array}{l}\text { Randomized controlled } \\
\text { trial }\end{array}$ & $\begin{array}{l}\text { Randomized controlled } \\
\text { trial }\end{array}$ \\
\hline $\begin{array}{l}\text { SL group (no. of } \\
\text { patients) }\end{array}$ & Leone F1000 (20) & 3M Smartclip (66) & 3M SmartClip (66) & 3M SmartClip (22) & Leone F1000 (20) & Ormco Damon Q (23) \\
\hline $\begin{array}{l}\text { CL group (no. of } \\
\text { patients) }\end{array}$ & Avex MX (20) & 3M Victory (71) & $3 \mathrm{M}$ Victory $(71)$ & 3M Gemini (24) & Avex MX (20) & $\begin{array}{l}\text { Rocky Mountain } \\
\text { Orthodontics Mini } \\
\text { Taurus (23) }\end{array}$ \\
\hline $\begin{array}{l}\text { Extraction or non- } \\
\text { extraction }\end{array}$ & Unreported & Both & Both & Non-extraction & Unreported & Unreported \\
\hline $\begin{array}{l}\text { Pretreatment mean age } \\
\text { (yrs.) }\end{array}$ & $\begin{array}{l}\text { F1000: } 14.7 \\
\text { Avex MX: } 14 \\
\text { Control: } 14.4\end{array}$ & 14 years 11 months & $\begin{array}{l}\text { SmartClip: } 15.5 \\
\text { Victory: } 14.5\end{array}$ & $\begin{array}{l}\text { SmartClip: } 15.48 \\
\text { Gemini: } 14.65\end{array}$ & $\begin{array}{l}\text { F1000: } 14.67 \\
\text { Avex MX: } 15.05\end{array}$ & $\begin{array}{l}\text { Damon Q:14.48 } \\
\text { Mini Taurus: } 13.30\end{array}$ \\
\hline Conclusions of Study & $\begin{array}{l}\text { No significant difference } \\
\text { was found between } \\
\text { SL and CL brackets } \\
\text { with metal ligatures } \\
\text { for periodontal status } \\
\text { and halitosis. Data was } \\
\text { obtained } 1 \text { week before } \\
\text { bonding, immediately } \\
\text { before bonding and at } 1 \text {, } \\
4 \text {, and } 8 \text { weeks. }\end{array}$ & $\begin{array}{l}\text { No significant difference } \\
\text { was found in regards } \\
\text { to overall treatment } \\
\text { time between SL and } \\
\text { CL bracket systems. } \\
\text { SPSS software with a } \\
\text { frequency histogram was } \\
\text { used for data analysis. } \\
\text { The use of PAR or ICON } \\
\text { was not reported. }\end{array}$ & $\begin{array}{l}\text { No significant } \\
\text { difference between } \\
\text { SL and CL brackets } \\
\text { in clinical discomfort } \\
\text { experienced at intervals } \\
\text { of } 1,3 \text {, and } 5 \text { days. }\end{array}$ & $\begin{array}{l}\text { No significant difference } \\
\text { was found in non- } \\
\text { extraction patients for } \\
\text { mandibular incisor } \\
\text { alignment between SL } \\
\text { and CL. }\end{array}$ & $\begin{array}{l}\text { No significant difference } \\
\text { was found between } \\
\text { SL and CL brackets } \\
\text { with metal ligation } \\
\text { for periodontal status } \\
\text { and colonization of } \\
\text { Streptococcus mutans } \\
\text { and Lactobacillus. Data } \\
\text { was recorded before } \\
\text { bonding and } 1 \text { month } \\
\text { afterward. }\end{array}$ & $\begin{array}{l}\text { SL brackets had } \\
\text { significantly reduced } \\
\text { halitosis and periodontal } \\
\text { indices compared to CL } \\
\text { brackets with elastomers. } \\
\text { PSL may prevent patients } \\
\text { from developing halitosis } \\
\text { and increase the ability to } \\
\text { maintain good hygiene. } \\
\text { Data was obtained before } \\
\text { bracket placement, and } \\
\text { at } 1 \text { and } 5 \text { weeks after } \\
\text { bonding. }\end{array}$ \\
\hline Bias Risk & Moderate & Low & Low & Low & Moderate & High \\
\hline
\end{tabular}

* RCT included in meta-analysis

Ormco $=$ SDS Ormco, $3 \mathrm{M}=3 \mathrm{M} /$ Unitek, $\mathrm{GAC}=$ Dentsply Sirona Ortho

Table 2b: Characteristics of RCTs included (and other quality investigations by publication date, See Appendix 2 for qualitative assessment)

\begin{tabular}{|c|c|c|c|c|c|c|}
\hline Author & Songra et al ${ }^{19}$ & Wong et a ${ }^{15}$ & Johansson, Lundstrom $^{31}$ & Alper $\mathrm{Oz}$ et al ${ }^{14}$ & Wahab et $\mathrm{al}^{20}$ & Mezomo et $\mathrm{a}^{11}$ \\
\hline Year & May 2014 & June 2013 & Sept 2012 & March 2012 & April 2011 & March 2011 \\
\hline Design & $\begin{array}{l}\text { Randomized controlled } \\
\text { trial* }\end{array}$ & $\begin{array}{l}\text { Randomized controlled } \\
\text { trial }\end{array}$ & $\begin{array}{l}\text { Randomized controlled } \\
\text { trial }\end{array}$ & $\begin{array}{l}\text { Randomized controlled } \\
\text { trial }\end{array}$ & $\begin{array}{l}\text { Randomized } \\
\text { controlled trial }\end{array}$ & $\begin{array}{l}\text { Randomized } \\
\text { controlled trial* }\end{array}$ \\
\hline $\begin{array}{l}\text { SL group (no. of } \\
\text { patients) }\end{array}$ & $\begin{array}{l}\text { Ormco Damon 3MX (41) } \\
\text { In-Ovation R (37) }\end{array}$ & Damon 3MX (14) & American Time2 (44) & 3M SmartClip (19) & Ormco Damon3 (14) & 3M SmartClip (15) \\
\hline $\begin{array}{l}\text { CL group (no. of } \\
\text { patients) }\end{array}$ & GAC Omni (20) & $\begin{array}{l}\text { Unreported American } \\
\text { CL with elastomeric } \\
\text { ligatures (13) } \\
\text { Unreported American } \\
\text { CL with Super Slick } \\
\text { elastomeric ligatures (13) }\end{array}$ & 3M Gemini (46) & 3M Mini Uni-Twin (19) & $\begin{array}{l}\text { Ormco MiniDiamond } \\
\text { (15) }\end{array}$ & 3M Gemini (15) \\
\hline $\begin{array}{l}\text { Extraction or } \\
\text { Non-extraction }\end{array}$ & Extraction & Extraction & Extraction & Extraction & Extraction & Extraction \\
\hline $\begin{array}{l}\text { Pretreatment mean age } \\
\text { (yrs.) }\end{array}$ & $11-18$ & $\begin{array}{l}\text { Damon 3MX: } 13.9 \\
\text { CL with elastomeric:14.1 } \\
\text { CL with Super Slick:13.7 }\end{array}$ & $\begin{array}{l}\text { Time2: } 15.3 \\
\text { Gemini: } 15.0\end{array}$ & 13.6 & $14-30$ & 18 \\
\hline $\begin{array}{l}\text { Conclusions } \\
\text { of Study }\end{array}$ & $\begin{array}{l}\text { Time to initial alignment } \\
\text { with four premolar } \\
\text { extractions was shown } \\
\text { to be statistically shorter } \\
\text { with CL than either } \\
\text { of the SL brackets } \\
\text { with different recall } \\
\text { intervals of } 6 \text { wks and } \\
12 \text { wks respectively. No } \\
\text { significant difference } \\
\text { in average response in } \\
\text { mm, was found between } \\
\text { active (ISL) and PSL } \\
\text { brackets. No statistical } \\
\text { difference was found } \\
\text { between the } 3 \text { bracket } \\
\text { types for space closure. }\end{array}$ & $\begin{array}{l}\text { The bracket-ligature type } \\
\text { was not found to affect } \\
\text { the rate of space closure. } \\
\text { Space closure involved } \\
\text { en masse movement of } \\
\text { the incisors and canines } \\
\text { against the premolars and } \\
\text { molars using coil springs. }\end{array}$ & $\begin{array}{l}\text { Overall treatment } \\
\text { time and number of } \\
\text { appointments was not } \\
\text { found to be significantly } \\
\text { different between SL and } \\
\text { CL. The ICON (Index of } \\
\text { Complexity, Outcome, } \\
\text { and Need) evaluation } \\
\text { was utilized to form and } \\
\text { analyze statistics. }\end{array}$ & $\begin{array}{l}\text { No significant difference } \\
\text { was found between SL } \\
\text { and CL groups in regards } \\
\text { to canine distalization } \\
\text { using rectangular arch } \\
\text { wire. TADs were used for } \\
\text { posterior anchorage. }\end{array}$ & $\begin{array}{l}\text { Tooth alignment in } \\
\text { the intial stage with } \\
\text { maxillary first premolar } \\
\text { extractions was generally } \\
\text { not higher for SL } \\
\text { compared to the CL } \\
\text { group over the first four } \\
\text { months of treatment. } \\
\text { However, CL alignment } \\
\text { in the first month was } \\
\text { higher than SL. CL } \\
\text { showed } 98 \% \text { of crowding } \\
\text { improvement compared } \\
\text { to } 67 \% \text { with SL after } \\
\text { four months using } \\
\text { irregularlity index. }\end{array}$ & $\begin{array}{l}\text { In vivo, there was no } \\
\text { difference in rate of space } \\
\text { closure found between } \\
\text { the SL and CL groups } \\
\text { for the distal movement } \\
\text { of upper canines and } \\
\text { mesial movement of } \\
\text { first molars. It was also } \\
\text { concluded that there was } \\
\text { less upper canine rotation } \\
\text { with SL SmartClip } \\
\text { compared to CL. }\end{array}$ \\
\hline Bias Risk & Low & Low & Low & Moderate & High & Moderate \\
\hline
\end{tabular}

*RCT included in meta-analysis

Ormco $=$ SDS Ormco, $3 \mathrm{M}=3 \mathrm{M} /$ Unitek, $\mathrm{GAC}=$ Dentsply Sirona Ortho 
Voudouris JC (2018) Self-ligation shortens chair time and compounds savings, with external bracket hygiene compared to conventional ligation: Systematic review with meta-analysis of randomized controlled trials

Table 2c. Characteristics of RCTs included (and other quality investigations by publication date, See Appendix 2 for qualitative assessment)

\begin{tabular}{|c|c|c|c|c|c|c|}
\hline Author & DiBiase et al $^{29}$ & Burrow $^{12}$ & Pandis et $\mathrm{al}^{51}$ & Miles and Weyant ${ }^{25}$ & Fleming et $\mathrm{al}^{17}$ & Pringle et $\mathrm{al}^{23}$ \\
\hline Year & February 2011 & September 2010 & June 2010 (e-pub 2009) & May 2010 & September 2009 & August 2009 \\
\hline Design & $\begin{array}{l}\text { Randomized controlled } \\
\text { trial }\end{array}$ & $\begin{array}{l}\text { Randomized controlled } \\
\text { trial* }\end{array}$ & $\begin{array}{l}\text { Prospective } \\
\text { cohort (completion of } \\
2007 \text { study portion) }\end{array}$ & $\begin{array}{l}\text { Randomized controlled } \\
\text { trial* }\end{array}$ & $\begin{array}{l}\text { Randomized controlled } \\
\text { trial (Part 2)* }\end{array}$ & $\begin{array}{l}\text { Randomized controlled } \\
\text { trial* }\end{array}$ \\
\hline $\begin{array}{l}\text { SL group (no. of } \\
\text { patients) }\end{array}$ & Ormco Damon3 (33) & $\begin{array}{l}\text { Ormco Damon3 (21),3M } \\
\text { Smartclip (22) }\end{array}$ & $\begin{array}{l}\text { Ormco Damon2 (27) } \\
\text { (mandibular incisor } \\
\text { torque }=-6^{\circ} \text { ) }\end{array}$ & In-Ovation C (34) & 3M SmartClip (29) & $\begin{array}{l}\text { Ormco Damon3 } \\
(24)\end{array}$ \\
\hline $\begin{array}{l}\text { CL group (no. of } \\
\text { patients) }\end{array}$ & Ormco Synthesis (29) & Victory Series (43) & $\begin{array}{l}\text { GAC Microarch (27) } \\
\text { (mandibular incisor } \\
\text { torque }=-1^{\circ} \text { ) }\end{array}$ & Clarity (34) & 3M Victory (31) & $\begin{array}{l}\text { Ormco Tru } \\
\text { Straight }(28)\end{array}$ \\
\hline $\begin{array}{l}\text { Extraction or Non- } \\
\text { extraction }\end{array}$ & Extraction & Extraction & Non-extraction & Non-extraction & Non-extraction & Extraction \\
\hline $\begin{array}{l}\text { Pretreatment mean age } \\
\text { (yrs.) }\end{array}$ & 16.27 & 14.8 & $\begin{array}{l}\text { Ormco Damon2: } 13.6 \text {, } \\
\text { GAC Microarch: } 13.9\end{array}$ & $\begin{array}{l}\text { In-Ovation C, Clarity: } \\
12-15\end{array}$ & $\begin{array}{l}\text { 3M SmartClip:16.32, } \\
\text { 3M Victory: } 16.37\end{array}$ & $\begin{array}{l}\text { Damon3: } 15.2 \\
\text { Tru straight: } 16.1\end{array}$ \\
\hline Conclusions of Study & $\begin{array}{l}\text { The results on lower } \\
\text { overall treatment time } \\
\text { were divided for SL } \\
\text { and CL groups. The } \\
\text { SL group was also not } \\
\text { found to reduce total } \\
\text { number of visits or result } \\
\text { in an improved occlusal } \\
\text { outcome using PAR } \\
\text { (Peer Assessment Rating) } \\
\text { scores in comparison to } \\
\text { the CL group. }\end{array}$ & $\begin{array}{l}\text { In vivo, the results } \\
\text { on rate of space closure } \\
\text { were divided for SL } \\
\text { and CL groups. The CL } \\
\text { group showed faster } \\
\text { distal movement of the } \\
\text { upper canines, possiblly } \\
\text { because of the narrow } \\
\text { bracket width of the SL } \\
\text { group. Narrow brackets } \\
\text { may bind with the } \\
\text { archwire or notch the } \\
\text { archwire. }\end{array}$ & $\begin{array}{l}\text { Both SL and CL } \\
\text { groups showed increases } \\
\text { in mandibular incisor } \\
\text { proclination and } \\
\text { intercanine width that } \\
\text { were not statistically } \\
\text { significant. There was } \\
\text { an additional intermolar } \\
\text { width increase after } \\
\text { treatment with SL group. }\end{array}$ & $\begin{array}{l}\text { Shortened chairtime was } \\
\text { found to be statistically } \\
\text { significant for SL using } \\
6 \text { anterior brackets } \\
\text { compared to CL. No } \\
\text { significant difference in } \\
\text { discomfort between SL } \\
\text { and CL brackets was } \\
\text { found at intervals of } 4 \\
\text { hours, } 1 \text { day, } 3 \text { days, and } \\
7 \text { days. }\end{array}$ & $\begin{array}{l}\text { Bracket type has } \\
\text { little to no effect on } \\
\text { incisor proclination } \\
\text { in non-extraction } \\
\text { patients. SL and CL } \\
\text { groups had similar } \\
\text { results. It was also } \\
\text { determined that bracket } \\
\text { type had little effect } \\
\text { on positional changes, } \\
\text { and inter-canine, inter- } \\
\text { first premolar, and } \\
\text { inter-second premolar } \\
\text { dimensions. More } \\
\text { incisor proclination } \\
\text { results from greater } \\
\text { alleviation of crowding. }\end{array}$ & $\begin{array}{l}\text { SL brackets result in } \\
\text { reduced intensity of } \\
\text { discomfort } \\
\text { compared to CL } \\
\text { brackets at } 12 \text { hour } \\
\text { intervals over } 7 \text { days. }\end{array}$ \\
\hline Bias Risk & Low & Moderate & Low & Moderate & Low & Low \\
\hline
\end{tabular}

* RCT included in meta-analysis

Ormco $=$ SDS Ormco, $3 \mathrm{M}=3 \mathrm{M} /$ Unitek, GAC=Dentsply Sirona Ortho

Table 2d. Characteristics of RCTs included (and other quality investigations by publication date, See Appendix 2 for qualitative assessment)

\begin{tabular}{|c|c|c|c|c|c|c|}
\hline Author & Fleming et $\mathrm{al}^{50}$ & Fleming et $\mathrm{al}^{24}$ & Scott et al ${ }^{18}$ & Jiang and $\mathrm{Fu}^{48}$ & Hamilton et $\mathrm{al}^{32}$ & Scott et $\mathrm{al}^{22}$ \\
\hline Year & May 2009 & January 2009 & October 2008 & August 2008 & July 2008 & March 2008 \\
\hline Design & $\begin{array}{l}\text { Randomized controlled } \\
\text { trial (Part 1) }\end{array}$ & $\begin{array}{l}\text { Randomized controlled } \\
\text { trial* }\end{array}$ & $\begin{array}{l}\text { Randomized } \\
\text { controlled trial* }\end{array}$ & Prospective cohort & Retrospective cohort & $\begin{array}{l}\text { Randomized controlled } \\
\text { trial* }\end{array}$ \\
\hline $\begin{array}{l}\text { SL group (no. of } \\
\text { patients) }\end{array}$ & 3M SmartClip (32) & SmartClip (26) & $\begin{array}{l}\text { Ormco Damon3 (32) } \\
\left(\text { mand. Inc torq }=-1^{\circ}\right)\end{array}$ & $\begin{array}{l}\text { Ormco Damon3 } \\
\left(\text { mand inc torq }=-1^{\circ}\right)\end{array}$ & GAC In-Ovation R (379) & Damon3 (33) \\
\hline $\begin{array}{l}\text { CL group (no. of } \\
\text { patients) }\end{array}$ & 3M Victory (33) & Victory (22) & $\begin{array}{l}\text { Ormco Synthesis }(28) \\
\text { (mand. Inc torq }=-1^{\circ} \text { ) }\end{array}$ & $\begin{array}{l}\text { CL metal preadjusted } \\
\text { brackets (13) (Shinya, } \\
\text { China) } \\
\left(\text { mand. Inc torq }=-1^{\circ} \text { ) }\right.\end{array}$ & 3M Victory (383) & Synthesis (29) \\
\hline $\begin{array}{l}\text { Extraction or Non- } \\
\text { extraction }\end{array}$ & Non-extraction & Extraction & Extraction & Non-extraction & Extraction & Extraction \\
\hline $\begin{array}{l}\text { Pretreatment mean age } \\
\text { (yrs.) }\end{array}$ & $\begin{array}{l}\text { 3M SmartClip: } 15.9,3 \mathrm{M} \\
\text { Victory: } 16.6\end{array}$ & $\begin{array}{l}\text { SmartClip: } 16.23 \\
\text { Victory: } 15.65\end{array}$ & $\begin{array}{l}\text { Ormco Damon3: } 16.2 \text {, } \\
\text { Synthesis: } 16.4\end{array}$ & $\begin{array}{l}\text { Ormco Damon3: } 14.5 \text {, } \\
\text { Conventional: } 15.3\end{array}$ & Unreported & 16 years 3 months \\
\hline Conclusions of Study & $\begin{array}{l}\text { SL systems were found } \\
\text { to be no more effective } \\
\text { at relieving alignment } \\
\text { irregularity than CL } \\
\text { systems in non-extraction } \\
\text { patients with mild } \\
\text { mandibular crowding. } \\
\text { A positive correlation } \\
\text { was found for improved } \\
\text { correction of irregularity } \\
\text { and level of pre-treament } \\
\text { irregularity. }\end{array}$ & $\begin{array}{l}\text { There was no } \\
\text { difference in discomfort } \\
\text { experienced between } \\
\text { SL and CL brackets at } \\
\text { intervals of } 4 \text { hours, } 24 \\
\text { hours, } 72 \text { hours, and } 7 \\
\text { days. }\end{array}$ & $\begin{array}{l}\text { No difference in } \\
\text { effectiveness for SL and } \\
\text { CL groups in init. overall } \\
\text { rate of mand. incisor } \\
\text { align \& mand premolar } \\
\text { extraction. Intermolar } \\
\text { width mainten increased. } \\
\text { Intercanine distance, } \\
\text { mild proclination of } \\
\text { mand. incisor and mild } \\
\text { arch length reduct for SL } \\
\text { and CL. No signif diff for } \\
\text { SL and CL groups. }\end{array}$ & $\begin{array}{l}\text { Increased lower incisor } \\
\text { proclination with both } \\
\text { SL and CL groups in } \\
\text { non extraction treatment } \\
\text { of mild crowding. SL } \\
\text { groups demonstrated } \\
\text { greater intermolar width } \\
\text { compared to CL group. }\end{array}$ & $\begin{array}{l}\text { SL showed greater } \\
\text { number of debonds } \\
\text { and other emergency } \\
\text { appointments. No } \\
\text { advantages for treatment } \\
\text { time, number of visits } \\
\text { and time for initial } \\
\text { alignment between SL } \\
\text { and CL. }\end{array}$ & $\begin{array}{l}\text { There were no significant } \\
\text { differences in perceived } \\
\text { discomfort at initial } \\
\text { tooth movement and } \\
\text { the discomfort did not } \\
\text { differ at subsequent } \\
\text { measurement times at } \\
\text { intervals of } 4 \text { hours, } 24 \\
\text { hours, } 3 \text { days, } 7 \text { days. }\end{array}$ \\
\hline Bias Risk & Low & Low & Low & High & Moderate & Low \\
\hline
\end{tabular}

*RCT included in meta-analysis

Ormco $=$ SDS Ormco, $3 \mathrm{M}=3 \mathrm{M} /$ Unitek, $\mathrm{GAC}=$ Dentsply Sirona Ortho 
Voudouris JC (2018) Self-ligation shortens chair time and compounds savings, with external bracket hygiene compared to conventional ligation: Systematic review with meta-analysis of randomized controlled trials

Table 2e. Characteristics of RCTs included (and other quality investigations by publication date, See Appendix 2 for qualitative assessment)

\begin{tabular}{|c|c|c|c|c|c|c|c|c|}
\hline Author & Paduano et $\mathrm{al}^{53}$ & Pandis et $\mathrm{al}^{16}$ & $\begin{array}{l}\text { Turnbull and } \\
\text { Birnie }^{60}\end{array}$ & Miles $^{40}$ & Miles et a ${ }^{41}$ & Pandis et $\mathrm{al}^{52}$ & Miles $^{42}$ & Eberting et $\mathrm{al}^{33}$ \\
\hline Year & $\begin{array}{l}2008 \text { (month) } \\
\text { unreported }\end{array}$ & August 2007 & March 2007 & April 2007 & May 2006 & January 2006 & November 2005 & November 2001 \\
\hline Design & Cross-sectional & $\begin{array}{l}\text { Randomized } \\
\text { controlled trial* }\end{array}$ & $\begin{array}{l}\text { Prospective Cross- } \\
\text { sectional }\end{array}$ & $\begin{array}{l}\text { Prospective cohort } \\
\text { (split mouth } \\
\text { design) }\end{array}$ & Prospective cohort & Prospective cohort & Prospective cohort & $\begin{array}{l}\text { Retrospective } \\
\text { cohort }\end{array}$ \\
\hline $\begin{array}{l}\text { SL group (no. of } \\
\text { patients) }\end{array}$ & $\begin{array}{l}\text { 3M SmartClip (10) } \\
\text { GAC In-Ovation } \\
\text { R (10) AO Time2 } \\
(10)\end{array}$ & $\begin{array}{l}\text { Ormco Damon2 } \\
(27) \text { (mand inc } \\
\left.\text { torq }=-6^{\circ}\right)\end{array}$ & $\begin{array}{l}\text { Ormco Damon2 } \\
(140)\end{array}$ & 3M SmartClip (14) & $\begin{array}{l}\text { Ormco Damon2 } \\
(58)\end{array}$ & $\begin{array}{l}\text { Ormco Damon2 } \\
(43)\end{array}$ & 3M SmartClip (29) & $\begin{array}{l}\text { Ormco Damon } \\
(108)\end{array}$ \\
\hline $\begin{array}{l}\text { CL group (no. of } \\
\text { patients) }\end{array}$ & $\begin{array}{l}\text { GAC Ovation } \\
\text { with STS ligatures } \\
\text { (10), GAC Ovation } \\
\text { with EL ligatures } \\
\text { (10) }\end{array}$ & $\begin{array}{l}\text { GAC Microarch } \\
(27)(\text { mand. inc } \\
\left.\text { torq }=-1^{\circ}\right)\end{array}$ & $\begin{array}{l}\text { Ormco Orthos } \\
(122)\end{array}$ & CL MBT twin (14) & $\begin{array}{l}\text { 3M Victory MBT } \\
(58)\end{array}$ & $\begin{array}{l}\text { GAC Microarch } \\
\text { (19) }\end{array}$ & $\begin{array}{l}\text { 3M Victory MBT } \\
(58)\end{array}$ & $\begin{array}{l}\text { CL Bracket not } \\
\text { indicated (107) }\end{array}$ \\
\hline $\begin{array}{l}\text { Extraction or } \\
\text { Non-extraction }\end{array}$ & Non-extraction & Non-extraction & Non-extraction & Extraction & Extraction & Non-extraction & Extraction & $\begin{array}{l}\text { Extraction and } \\
\text { Non-extraction }\end{array}$ \\
\hline $\begin{array}{l}\text { Pretreatment } \\
\text { mean age (yrs.) }\end{array}$ & $\begin{array}{l}\text { Unreported } 12-30 \\
\text { range }\end{array}$ & $\begin{array}{l}\text { Ormco Damon2: } \\
\text { 13.5, GAC } \\
\text { Microarch: } 13.9\end{array}$ & $\begin{array}{l}\text { Ormco Damon2: } \\
13.7 \text {, } \\
\text { Ormco Orthos: } \\
14.4\end{array}$ & 13.1 (median) & 16.3 & 14 & 17.1 & Unreported \\
\hline $\begin{array}{l}\text { Conclusions of } \\
\text { Study }\end{array}$ & $\begin{array}{l}\text { SL showed more } \\
\text { efficient arch } \\
\text { wire removal and } \\
\text { placement late in } \\
\text { the orthodontic } \\
\text { treatment. Type } \\
\text { of SL bracket } \\
\text { determined } \\
\text { ligation time in the } \\
\text { mandibular arch. }\end{array}$ & $\begin{array}{l}\text { No statistical } \\
\text { difference } \\
\text { between SL and } \\
\text { CL groups for } \\
\text { overall increases } \\
\text { in mandibular } \\
\text { proclination and } \\
\text { intercanine width } \\
\text { treated non- } \\
\text { extraction. For } \\
\text { irregularity index } \\
<5, \text { SL was } 2.7 \mathrm{x} \\
\text { faster and showed } \\
\text { a statistically } \\
\text { higher increase in } \\
\text { intermolar width. } \\
\text { However, there } \\
\text { were no significant } \\
\text { differences in } \\
\text { time required to } \\
\text { align mandibular } \\
\text { crowding between } \\
\text { the SL and CL. }\end{array}$ & $\begin{array}{l}\text { SL demonstrated } \\
\text { significantly lower } \\
\text { mean archwire } \\
\text { ligation time for } \\
\text { both placing and } \\
\text { removing arch } \\
\text { wires. }\end{array}$ & $\begin{array}{l}\text { SL and CL with } \\
\text { metal ligation } \\
\text { groups showed } \\
\text { no significant } \\
\text { difference in rate } \\
\text { of en masse space } \\
\text { closure. }\end{array}$ & $\begin{array}{l}\text { SL had a higher } \\
\text { bracket failure. } \\
\text { SL and CL } \\
\text { groups showed no } \\
\text { difference during } \\
\text { initial alignment. }\end{array}$ & $\begin{array}{l}\text { SL and edgewise } \\
\text { brackets showed } \\
\text { no significant } \\
\text { difference in failure } \\
\text { incidence using } \\
\text { self-etching primer } \\
\text { or acid-etching. }\end{array}$ & $\begin{array}{l}\text { No difference } \\
\text { between SL and CL } \\
\text { groups for reducing } \\
\text { initial stage } \\
\text { irregularity. }\end{array}$ & $\begin{array}{l}\text { SL group showed } \\
\text { significantly } \\
\text { lower treatment } \\
\text { times than CL. } \\
\text { It appeared that } \\
\text { SL group had } \\
\text { significantly higher } \\
\text { ABO scores than } \\
\text { those treated with } \\
\text { CL edgewise } \\
\text { brackets. }\end{array}$ \\
\hline Bias Risk & High & Low & Moderate & Moderate & Moderate & Moderate & Moderate & High \\
\hline
\end{tabular}

*RCT included in meta-analysis

Ormco $=$ SDS Ormco, $3 \mathrm{M}=3 \mathrm{M} /$ Unitek, $\mathrm{GAC}=$ Dentsply Sirona Ortho

Table 2f. Characteristics of RCTs included (and other quality investigations by publication date, See Appendix 2 for qualitative assessment)

\begin{tabular}{|c|c|c|c|}
\hline Author & Harradine $^{13}$ & Berger and Byloff ${ }^{54}$ & Maijer and Smith ${ }^{55}$ \\
\hline Year & November 2001 & May 2001 & January 1990 \\
\hline Design & Retrospective cohort & Cross-sectional & Cross-sectional \\
\hline SL group (no. of patients) & $\begin{array}{l}\text { Ormco Damon SL (30), Chair time study } \\
\mathrm{n}=50 \text {, Bracket complications study } \mathrm{n}=25\end{array}$ & $\begin{array}{l}\text { Strite SPEED (20), Ormco Damon,Adenta } \\
\text { Time, Ormco Twinlock }\end{array}$ & "A" Company Activa (14) \\
\hline CL group (no. of patients) & $\begin{array}{l}\text { Type not specified (30), Chair time } \\
\text { study } n=50 \text {, Bracket complications study } \\
n=25\end{array}$ & Ormco Mini-twin (40) & "A" Company SWA brackets (14) \\
\hline Extraction or Non-extraction & Extraction and Non-extraction & Non-extraction & Unreported \\
\hline Pretreatment mean age (yrs.) & Unreported & Unreported & Unreported \\
\hline Conclusions of Study & $\begin{array}{l}\text { SL group showed } \\
\text { four months shorter treatment time and } \\
\text { needed four fewer visits on average } \\
\text { compared to CL group. Both groups } \\
\text { produced equal and good reduction in } \\
\text { occlusal irregularity. SL opening and closing } \\
\text { chair times were significantly shorter with SL } \\
\text { compared to CL group. }\end{array}$ & $\begin{array}{l}\text { Four different SL } \\
\text { brackets showed significantly lower total } \\
\text { opening and closing times than CL. Damon } \\
\text { needed most time and SPEED least time to } \\
\text { open and close. }\end{array}$ & $\begin{array}{l}\text { SL brackets had a significantly shortened } \\
\text { chair time advantage compared to CL } \\
\text { brackets }\end{array}$ \\
\hline Bias Risk & Moderate & High & High \\
\hline
\end{tabular}

* RCT included in meta-analysis

Ormco $=$ SDS Ormco, $3 \mathrm{M}=3 \mathrm{M} /$ Unitek, GAC $=$ Dentsply Sirona Ortho 
Voudouris JC (2018) Self-ligation shortens chair time and compounds savings, with external bracket hygiene compared to conventional ligation: Systematic review with meta-analysis of randomized controlled trials

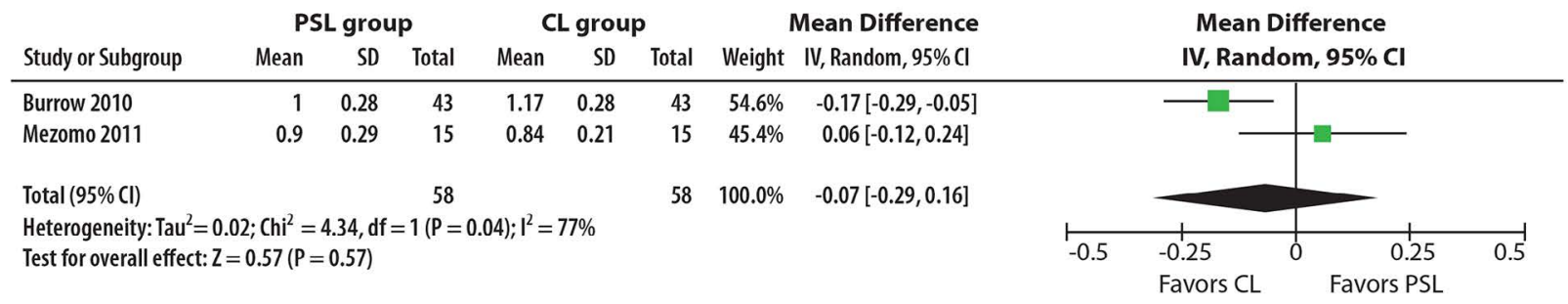

Figure 2. Forest plot of in vivo rate of space closure of two RCTS for meta-analysis (mm)

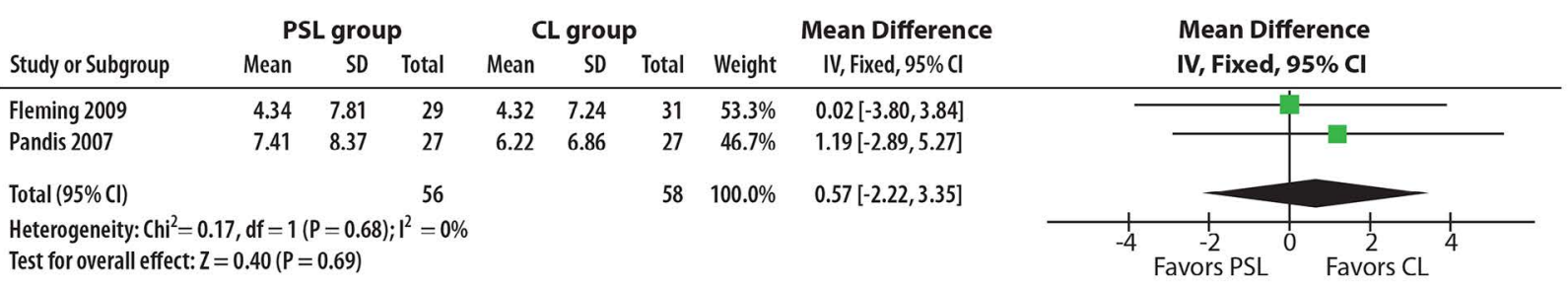

Figure 3. Forest plot of incisor proclination of two RCTS for meta-analysis (degrees)

\begin{tabular}{|c|c|c|c|c|c|c|c|c|c|}
\hline \multirow[b]{2}{*}{ Study or Subgroup } & \multicolumn{3}{|c|}{ PSL group } & \multicolumn{3}{|c|}{ CL group } & \multicolumn{2}{|c|}{ Std. Mean Difference } & \multirow{2}{*}{$\begin{array}{l}\text { Std. Mean Difference } \\
\text { IV, Random, } 95 \% \mathrm{CI}\end{array}$} \\
\hline & Mean & SD & Total & Mean & SD & Total & Weight & IV, Random, 95\% CI & \\
\hline Scott 2008 & 253 & 63.6 & 32 & 243 & 42.5 & 28 & $51.5 \%$ & $10.00[-17.08,37.08]$ & \\
\hline Songra 2014 & 422 & 124 & 41 & 251 & 107 & 20 & $48.5 \%$ & $171.00[110.67,231.33]$ & \\
\hline Total $(95 \% \mathrm{Cl})$ & & & 73 & & & 48 & $100.0 \%$ & $88.15[-69.56,245.86]$ & \\
\hline \multicolumn{9}{|c|}{$\begin{array}{l}\text { Heterogeneity: } \text { Tau }^{2}=12391.31 ; \mathrm{Chi}^{2}=22.77, \mathrm{df}=1(\mathrm{P}<0.00001) ; \mathrm{I}^{2}=96 \% \\
\text { Test for overall effect: } \mathrm{Z}=1.10(\mathrm{P}=0.27)\end{array}$} & 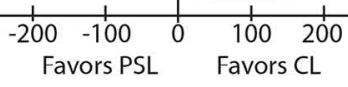 \\
\hline
\end{tabular}

Figure 4. Forest plot of rate of mandibular alignment in days of two RCTS for meta-analysis (mm)

made no practical, clinical difference to a patient's treatment [5] and the MA also demonstrated no statistically significant difference. There was significant heterogeneity in studies on space closure. One RCT [6] investigated used large rectangular archwire rather than round archwire $[3,4]$ and skeletal anchorage of TADs, and another RCT [7] used en masse movement of incisors and canines with a continuous arch where both RCTs did not allow inclusion of this data for MA.

\section{Reduced incisor proclination}

The two RCTs, by Pandis, et al. [8] and Fleming, et al. [9] were included since they used non-extraction to assess incisor proclination (Figure 3), but the differences were not found to be statistically significant. Another RCT [10] was not included because it studied incisor proclination with extraction treatment and moderate dental crowding.

\section{Rate of initial mandibular incisor alignment (in days) in standardized mean difference}

The RCT by Songra, et al. [11] and the data from Scott [10] including mandibular premolar extractions were used for MA, toward the construction of a forest plot (Figure 4). The results showed in the early alignment stage the difference was not statistically significant. The overall effect must be interpreted with reservation since the $\mathrm{I}^{2}$ value was high, due to the heterogeneous methodology, where age ranges and time intervals were different [11]. However, the two RCTs were the highest quality studies currently available with continuity because both investigated mandibular incisor alignment with mandibular premolar extractions, and SL brackets with a control. One other RCT [12] used upper premolar extractions and had a high risk of bias, while another RCT [13] included non-extraction patients precluding MA.

\section{Initial discomfort}

Four RCTs [14-17] were found which had an acceptable risk of low to moderate bias and studied discomfort after 4 hours, 24 hours, 3 days and 7 days following archwire insertion and had reported a MA. They employed the VAS (Visual Analog Scale) assessment and found no differences in discomfort between PSL and CL. Another multi-centered RCT [18] compared PSL and CL at 24 hours and 3 days but this data was based on the VRS (Verbal Rating Scale) that produced inaccuracies since it relied on verbal responses compared to the tangible, visual analog scale, that precluded MA with the other 4 RCTs.

\section{Overall treatment time}

Three RCTs [19-21] on total treatment time with low risk of bias have been reported. None of these revealed any statistically significant differences in total treatment time between SL and CL. Other retrospective studies $[5,22,23]$ investigated treatment time (and also used occlusal indices) that may have suffered from a higher risk of bias [1]. The significant, heterogeneous designs of these 3 RCTs above also required the use of standardized mean differences to minimize methodological differences among several investigations of overall treatment time [9,24-27]. In terms of assessing potential reduction in overall treatment time, results could not be synthesized with the three RCTs above for MA because of the different statistical analyses and ratings systems used (PAR, ICON, SPSS). Although PAR and ICON scores are not measures of treatment time, DiBiase, et al. [19] used PAR (Peer Assessment Rating) scores, O’Dwyer, et al. [20] used SPSS (statistical package for the social sciences, IBM Corporation) software with a frequency histogram and a different rating system, while Johansson and Lundstrom [21] used ICON (Index of Complexity, Outcome, and Need) evaluation. Consequently, a reduction in overall treatment time could not be reliably determined. 
Voudouris JC (2018) Self-ligation shortens chair time and compounds savings, with external bracket hygiene compared to conventional ligation: Systematic review with meta-analysis of randomized controlled trials

\section{Halitosis and periodontal indices}

One RCT by Nalcaci, et al. [28] found halitosis and periodontal indices were significantly increased for CL with elastomers compared to PSL. Another RCT [29] used CL with metal ligatures studying similar parameters and found no differences. However, the two different CL methods, did not permit MA. The data from a third RCT [30] studied periodontal indices and bacterial levels and this data also could not be synthesized for MA

\section{Shortened chair time}

One RCT by Miles and Weyant [17] found shortened chair time that was statistically and clinically significant of 130.2 secs/arch saved compared to metal CL, using six anterior esthetic brackets.

\section{Discussion}

This SR applied additional, high quality RCTs available for MA [1]. The synthesis of data from RCTs was possible for several clinical claims regarding SL in this SR. The electronic search led to the selection of 236 articles (Appendix 3). All were published in English except one, which was in Chinese [31] that were translated for use in this review. The final 35 SL studies expanded the qualitative analysis [3-26,28-38]. Due to the low number of RCT studies for each outcome, implications could not be derived from funnel plots to assess publication bias.

\section{Rate of in vivo space closure}

The great majority of SL brackets in the included RCTs were the PSL type, and additional interactive (active) SL studies were clearly needed. Space closure with narrower PSL brackets [3] can result in binding and notching of the archwire with conventional retraction forces that may have been higher for the narrower brackets compared to $\mathrm{CL}$ brackets. In the RCTs of this SR, the PSL brackets would have allowed a looser fit of the archwire in the slot compared to CL, greater tipping and consequently greater resistance to sliding. Other factors may have also affected space closure including bone density, occlusion, or dental interferences.

\section{Reduced incisor proclination, rate of initial mandibular incisor alignment and discomfort}

The NRT [31] for incisor proclination was not used in this SR because it had a high risk of bias. Although it was a non-extraction study, it also used only mild dental crowding $(3 \mathrm{~mm})$ compared to an RCT [10] using extraction with moderate crowding. Due to the statistically insignificant difference found, the clinical relevance of incisor proclination requires further study [1].

Initial mandibular incisor alignment may have been limited because PSL brackets do not seat archwires into the base of the slot. More interactive SL studies are needed since they are designed to seat the archwire earlier than PSL, used in one RCT [11] although there was a concern for consistency in methods because CL brackets were evaluated every 6 weeks compared to SLs evaluated every 12 weeks. The RCT by Fleming, et al. [32] measured rate of alignment but was excluded in this MA because it used non-extraction treatment and the irregularity index rather than the measurement in days. Discomfort is a complex outcome to evaluate in patients because it is highly subjective with wide ranges of sensitivities and perceptions of discomfort. RCTs on discomfort can sometimes employ different scales such as the verbal rating scale (VRS) [18] rather than a visual analog scales (VAS) [1417] although both can offer imprecise results. Contradictory results on discomfort can also be related to other factors such as age and gender.

\section{Shortened chair time}

From the electronic search, the finding by Miles and Weyant [17] of shortened chair time for six anterior, esthetic, SL brackets of $130.2 \mathrm{sec}$, or 2.2 minutes/ $(1 \mathrm{arch})$ that was statistically and clinically significant compared to metal CL in this one RCT reported, was also found in the majority of studies examined [5,27,35-37, and Appendix 4]. There was also support for the above RCT in the study by Turnbull and Birnie [38] (Appendix 2b). They demonstrated statistically significant SL clip closing $(\mathrm{P}<.001)$ and opening $(\mathrm{P}<.01)$ compared to elastomeric $\mathrm{CL}$ with a total SL chair time savings of $76.8 \mathrm{sec} / \mathrm{arch}$, or $2.5 \mathrm{~min} /(2 \mathrm{arches})$ working with more posterior brackets (10 brackets/arch). Although archwire ligation is one of the most repetitive orthodontic procedures and clinical techniques vary, the compounding effect [27] of SL chair time savings of the lower $2.5 \mathrm{~min} /$ patient [38] was calculated. Applying 40 patients/ $8 \mathrm{hr}$-day, 4 days/wk produced an interesting potential savings of $102.4 \mathrm{~min} /$ day or 1.7 hours/day. Compounded chair time savings were similarly confirmed when the conclusions of Chen, et al., 2000 were applied (Figures 5A and 5B).

Physically, external bracket hygiene for 20 brackets/patient, distinguished from patient oral hygeine, was also logically improved by the ligature-free nature of SL brackets (Figure 6). SL eliminated longterm repetitive errors discussed in industrial engineering by replacing 20 external ligatures/patient

A.

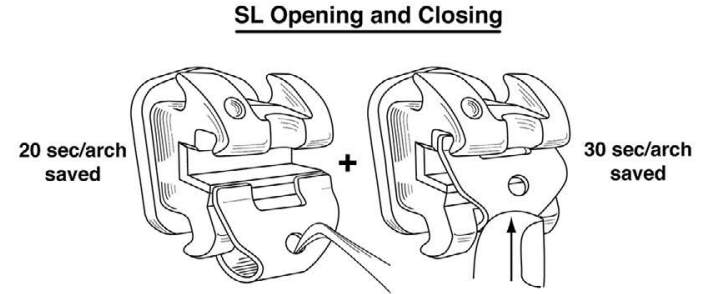

$=50 \mathrm{sec} /$ arch saved $\times 2$ arches/patient $=100 \mathrm{sec} /$ patient saved (Chen et al below

Compounded statistical chair time savings with self-ligation* (compared to conventional ligation)

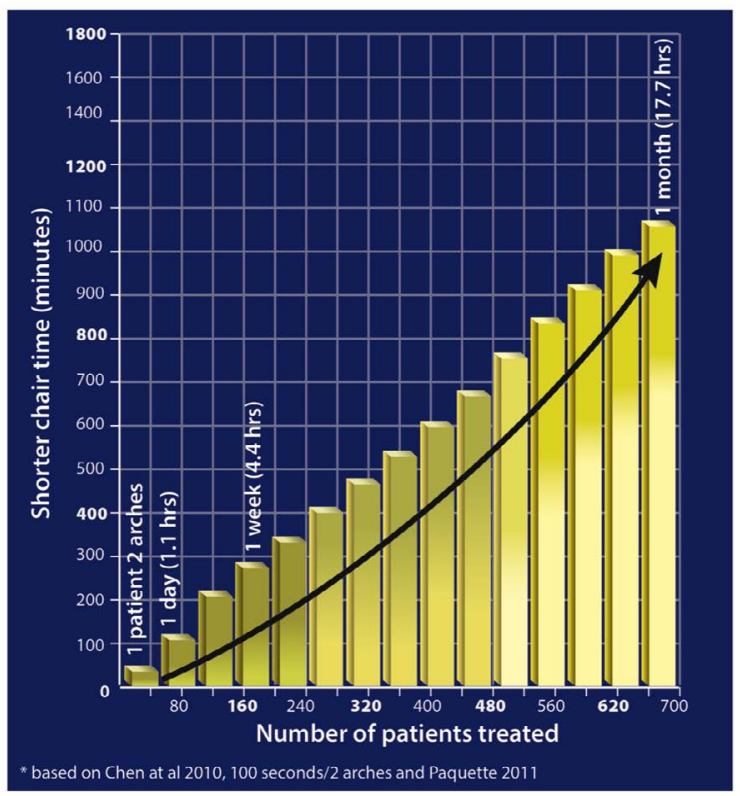

Figure 5. A) SL ligature-free opening, and closing appears to contribute to statistically shortened chair time. B) Demonstrates the compounding effect of the shortened chair time with SL in a medium-size practice treating 40 patients/day, 4 days/week over one month (17.7 hrs) 
Voudouris JC (2018) Self-ligation shortens chair time and compounds savings, with external bracket hygiene compared to conventional ligation: Systematic review with meta-analysis of randomized controlled trials

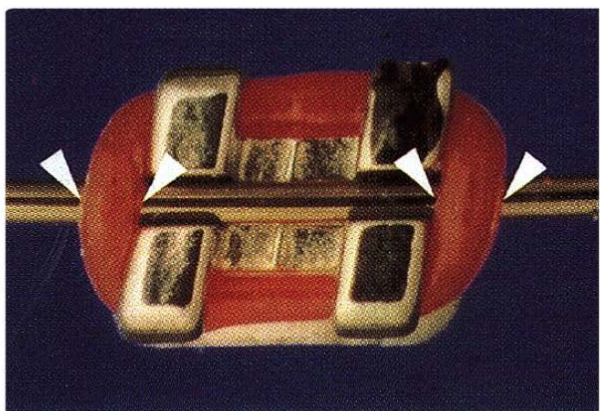

Conventional Ligation X 20/patient poor external bracket hygiene

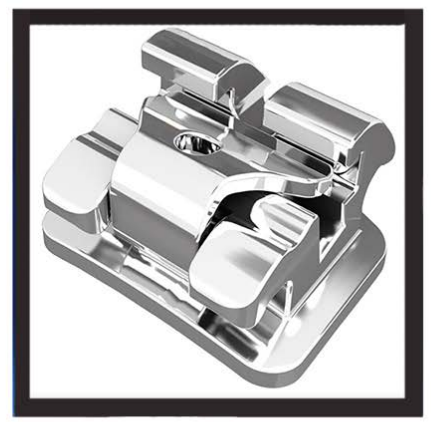

Self-ligation (ligature-free) external bracket hygiene

Figure 6. Comparison of conventional ligation (CL) external bracket hygiene confounded by decaying, distorting and hydrolytically decomposing elastomers X 20/patient repeatedly compared to SL (mini Prevail ${ }^{\mathrm{TM}}$ ) without elastomers

\section{Conclusions}

Rigorous, high standards in a systematic review were used including RCTs to study passive SL clinical claims and found:

1. Shortened chair time for SL was found in both one RCT, a good prospective, cross-sectional study and all five of the other investigations on chairtime efficiency. One further RCT would also allow for meta-analysis. When examining other outcomes, shortened chairtime appears to be one of the main reasons for the relatively high frequency of SL application by clinicians currently.

2. The logical question of whether the external hygiene of 20 brackets/ patient was improved by SL with the removal of the 20 decaying and hydrolytically decomposing elastomers/patient after one month intraorally, eliminated by ligature-free SL, was another real advantage for clinicians. Multiple bracket hygiene was different, than unpredictable patients' oral hygiene.
3. Faster in vivo space closure during upper canine retraction into first premolar extraction sites, was not statistically significant, or clinically significant.

4. Reduced incisor proclination showed the effective amount was not statistically significant.

5. Reduced number of days for mandibular incisor alignment was statistically insignificant, with clinically insignificant differences.

6. Initial discomfort was highly subjective, and no differences could be found using four RCTs and meta-analysis.

7. Total treatment time, similar to halitosis, was not found to be different in three unmatched RCTs that precluded meta-analysis.

More well-conducted RCT's, and particularly of interactive SL are indicated because the majority study PSL (Table 3).

Table 3. Six cohorts of SL appliances

\begin{tabular}{|c|c|c|c|c|c|}
\hline Metal ISL (twin) & Ceramic ISL (twin) & Metal PSL & Ceramic (or Clear) PSL & Metal ISL (single) & Lingual SL \\
\hline Empower 2009 (AO) & Empower Clear 2012 (AO) & $\begin{array}{l}\text { Damon SL, 2, 3, MX } 1998 \\
\text { (Ormco) }\end{array}$ & Damon Aesth 2009 (Ormco) & Speed 1980 (Strite) & In-Ovation L 2006 (DSO) \\
\hline Experience Mini 2014 (GCOA) & Experience C 2014 (GCOA) & $\begin{array}{l}\text { Carriere SLX } 2004 \text { (Hen Schein } \\
\text { OO) }\end{array}$ & $\begin{array}{l}\text { Carriere SLX 3D } 2016 \text { (Hen } \\
\text { Schein OO) }\end{array}$ & Time 1994 (Adenta) & $\begin{array}{l}\text { Evolution LT } 2002 \\
\text { (Adenta) }\end{array}$ \\
\hline $\begin{array}{l}\text { In-Ovation-R 2000, -X } 2017 \\
\text { (DSO) }\end{array}$ & In-Ovation C 2006 (DSO) & Smartclip SL, 22004 (3M) & Clarity SL 2007 (3M) & & Harmony 2012 (AO) \\
\hline $\begin{array}{l}\text { Sensation M } 2010 \text { (Hen Schein } \\
\text { OT) }\end{array}$ & $\begin{array}{l}\text { Sensation C } 2012 \text { (Hen Schein } \\
\text { OT) }\end{array}$ & Opal Metal 2006 (Ultradent) & Opal 2004 (Ultradent) & & \\
\hline $\begin{array}{l}\text { ZIP SL, LP } 2014 \text { (SIA Orth } \\
\text { Manufact) }\end{array}$ & dinamique c 2016 (Dentaurum) & BioPassive 3G 2010 (Forestad) & Oyster 2001 (Gestenco) & & \\
\hline mini Prevail 2017 (G\&H) & Krystal SL 2016 (Dynaflex) & Lotus 2008 (Henry Schein OT) & $\begin{array}{l}\text { Velocity SLB C } 2017 \\
\text { (Lancer) }\end{array}$ & & \\
\hline Endurance 2017 (Dynaflex) & QuickKlear 2G 2012 (Forestad) & Vision LP 2007 (AO) & Ascend SL C 2016 (RMO) & & \\
\hline Velocity SLB 2016 (Lancer) & Nexus Clear 2010 (Ormco) & Agility 2010 (ODP) & & & \\
\hline dinamique m 2016 (Dentaurum) & & Tenbrooke T1 2009 (Ortho Classic) & & & \\
\hline Alpine SL 2017 (RMO) & & Forces 2011 (Leone) & & & \\
\hline Quick 2006 (Forestad) & & FLI SL 2012, Altitude 2016 (RMO) & & & \\
\hline Nexus 2010 (Ormco) & & Glide 2006, Velocity 2016 (Lancer) & & & \\
\hline BioQuick 3G 2010 (Forestad) & & $\begin{array}{l}\text { Activa } 1986 \text { ("A" Company/ } \\
\text { Ormco) }\end{array}$ & & & \\
\hline Victory SL 2014 (3M) & & Mobi-lok 1980 (Forestad) & & & \\
\hline \multirow[t]{3}{*}{$\begin{array}{l}\text { Lotus Active } 2015 \text { (Henry Schein } \\
\text { OT) }\end{array}$} & & Edgelok 1972 (Ormco) & & & \\
\hline & & Ford-lock 1942 (RMO) & & & \\
\hline & & Russell Lock 1935 (N/A) & & & \\
\hline
\end{tabular}

Ormco=SDS Ormco, 3M=3M/Unitek, DSO=Dentsply Sirona Ortho, AO=American Orthodontics, OT=Ortho Technologies, OO=Ortho Organizers, RMO=Rocky Mountain Orthodontics, Forestad=Forestadent, $\mathrm{GCOA}=\mathrm{GC}$ Orthodontics America, $\mathrm{C}=$ Ceramic (some estimates of introduction $\mathrm{yr}$ ) 
Voudouris JC (2018) Self-ligation shortens chair time and compounds savings, with external bracket hygiene compared to conventional ligation: Systematic review with meta-analysis of randomized controlled trials

\section{References}

1. Kaklamanos EG, Athanasiou AE (2011) Systematic review of self-ligating brackets. Am J Orthod Dentofacial Orthop 139: 145-146. [Crossref]

2. Viechtbauer W (2010) Conducting meta-analyses in $\mathrm{R}$ with the metafor package. $J$ Statistical Software 36: 1-48.

3. Burrow SJ (2010) Canine retraction rate with self-ligating brackets vs conventional edgewise brackets. Angle Orthod 80: 626-633. [Crossref]

4. Mezomo M, de Lima ES, de Menezes LM, Weissheimer A, Allgayer S (2011) Maxillary canine retraction with self-ligating and conventional brackets. Angle Orthod 81: 926929.

5. Harradine NW (2001) Self-ligating brackets and treatment efficiency. Clin Orthod Res 4: 220-227. [Crossref]

6. Alper Oz A, Arici N, Arici S (2012) The clinical and laboratory effects of bracket type during canine distalization with sliding mechanics. Angle Orthodontist 82: 326-332.

7. Wong H, Collins J, Tinsley D, Sandler J, Benson P (2013) Does the bracket-ligature combination affect the amount of orthodontic space closure over three months? A randomized controlled trial. J Orthod 40: 155-162. [Crossref]

8. Pandis N, Polychronopoulou A, Eliades T (2007) Self-ligating vs conventional brackets in the treatment of mandibular crowding: a prospective clinical trial of treatmen duration and dental effects. Am J Orthod Dentofacial Orthop 132: 208-215. [Crossref]

9. Fleming PS, DiBiase AT, Sarri G, Lee RT (2009) Comparison of mandibular arch changes during alignment and leveling with 2 preadjusted edgewise appliances. $\mathrm{Am} \mathrm{J}$ Orthod Dentofacial Orthop 136: 340-347. [Crossref]

10. Scott P, DiBiase AT, Sherriff M, Cobourne MT (2008) Alignment efficiency of Damon 3 self-ligating and conventional orthodontic bracket systems: a randomized clinical trial. Am J Orthod Dentofacial Orthop 134: 470.e1-8. [Crossref]

11. Songra G, Clover M2, Atack NE3, Ewings P4, Sherriff M5, et al. (2014) Comparative assessment of alignment efficiency and space closure of active and passive self-ligating vs conventional appliances in adolescents: a single-center randomized controlled trial. Am J Orthod Dentofacial Orthop 145: 569-578. [Crossref]

12. Wahab RM, Idris H, Yacob H, Ariffin SH (2012) Comparison of self- and conventionalligating brackets in the alignment stage. Eur J Orthod 34: 176-181. [Crossref]

13. Celikoglu M, Bayram M, Nur M, Kilkis D (2015) Mandibular changes during initial alignment with SmartClip self-ligating and conventional brackets: A single-center prospective randomized controlled clinical trial. Korean J Orthod 45: 89-94. [Crossref]

14. Scott P, Sherriff M, Dibiase AT, Cobourne MT (2008) Perception of discomfort during initial orthodontic tooth alignment using a self-ligating or conventional bracket system: a randomized clinical trial. Eur J Orthod 30: 227-232. [Crossref]

15. Pringle AM, Petrie A, Cunningham SJ, McKnight M (2009) Prospective randomized clinical trial to compare pain levels associated with 2 orthodontic fixed bracket systems. Am J Orthod Dentofacial Orthop 136: 160-167. [Crossref]

16. Fleming PS, DiBiase AT, Sarri G, Lee RT (2009) Pain experience during initia alignment with a self- ligating and a conventional fixed orthodontic appliance system: a randomized controlled clinical trial. Angle Orthod 79: 46-50.

17. Miles P, Weyant R (2010) Porcelain brackets during initial alignment: are self-ligating cosmetic brackets more efficient? Aust Orthod J 26: 21-26. [Crossref]

18. Rahman S, Spencer RJ, Littlewood SJ, O’Dywer L, Barber SK, Russell JS (2015) A multicenter randomized controlled trial to compare a self-ligating bracket with a conventional bracket in a UK population: Part 2: Pain perception. Angle Orthod.
19. DiBiase AT, Nasr IH, Scott P, Cobourne MT (2011) Duration of treatment and occlusa outcome using Damon 3 self-ligated and conventional orthodontic bracket systems in extraction patients: a prospective randomized clinical trial. Am J Orthod Dentofacial Orthop 139: e111-e116. [Crossref]

20. O’Dwyer L, Littlewood SJ, Rahman S, Spencer RJ, Barber SK, Russell JS (2015) A multi-center randomized controlled trial to compare a self-ligating bracket with a conventional bracket in a UK population: Part 1: treatment efficiency. Angle Orthod.

21. Johansson K, Lundström F (2012) Orthodontic treatment efficiency with self-ligating and conventional edgewise twin brackets: a prospective randomized clinical trial Angle Orthod 82: 929-934. [Crossref]

22. Hamilton R, Goonewardene MS, Murray K (2008) Comparison of active self-ligating brackets and conventional pre-adjusted brackets. Aust Orthod J 24: 102-109. [Crossref]

23. Eberting JJ, Straja SR, Tuncay OC (2001) Treatment time, outcome, and patient satisfaction comparisons of Damon and conventional brackets. Clin Orthod Res 4: 228 234. [Crossref]

24. Miles PG (2007) Self-ligating vs conventional twin brackets during en-masse space closure with sliding mechanics. Am J Orthod Dentofacial Orthop 132: 223-225. [Crossref]

25. Miles PG, Weyant RJ, Rustveld L (2006) A clinical trial of Damon 2 vs conventional twin brackets during initial alignment. Angle Orthod 76: 480-485. [Crossref]

26. Miles PG (2005) SmartClip versus conventional twin brackets for initial alignment: is there a difference? Aust Orthod J 21: 123-127. [Crossref]

27. Paquette DE (2011) Biased look at self-ligation. Am J Orthod Dentofacial Orthop 139 574. [Crossref]

28. Nalçaci R, Özat Y, Çokakoglu S, T"rkkahraman H, Önal S, Kaya S (2014) Effect of bracket type on halitosis, periodontal status, and microbial colonization. Angle Orthod 84: 479-485. [Crossref]

29. Kaygisiz E, Uzuner FD, Yuksel S, Taner L, Ã ulhaoÄŸlu R, et al. (2015) Effects of self-ligating and conventional brackets on halitosis and periodontal conditions. Angle Orthod 85: 468-473. [Crossref]

30. Uzuner FD, Kaygisiz E, Cankaya ZT (2014) Effect of the bracket types on microbial colonization and periodontal status. Angle Orthod 84: 1062-1067. [Crossref]

31. Jiang RP, Fu MK (2008) Non-extraction treatment with self-ligating and conventional brackets. Zhonghua Kou Qiang Yi Xue Za Zhi 43: 459-463.

32. Fleming PS, DiBiase AT, Sarri G, Lee RT (2009) Efficiency of mandibular arch alignment with 2 preadjusted edgewise appliances. Am J Orthod Dentofacial Orthop 135: 597-602. [Crossref]

33. Pandis NP, Polychronopoulou A, Makou M, Eliades T (2010) Mandibular dental arch changes associated with treatment of crowding using self-ligating and conventional brackets. Eur J Orthod 32: 248-253. [Crossref]

34. Pandis N, Polychronopoulou A, Eliades T (2006) Failure rate of self-ligating and edgewise brackets bonded with conventional acid etching and a self-etching primer: a prospective in vivo study. Angle Orthod 76: 119-122. [Crossref]

35. Paduano S, Cioffi I, Iodice G, Rapuano A, Silva R (2008) Time efficiency of selfligating vs conventional brackets in orthodontics: effect of appliances and ligating systems. Prog Orthod 9: 74-80. [Crossref]

36. Berger J, Byloff FK (2001) The clinical efficiency of self-ligated brackets. $J$ Clin Orthod 35: 304-308. [Crossref]

37. Maijer R, Smith DC (1990) Time savings with self-ligating brackets. J Clin Orthod 24 29-31. [Crossref]

38. Turnbull NR, Birnie DJ (2007) Treatment efficiency of conventional vs self-ligating brackets: effects of archwire size and material. Am J Orthod Dentofacial Orthop 131 395-399. [Crossref]

Copyright: (C2018 Voudouris JC. This is an open-access article distributed under the terms of the Creative Commons Attribution License, which permits unrestricted use, distribution, and reproduction in any medium, provided the original author and source are credited. 